\title{
Correction to: Object Manipulation and Tool Use in Nicobar Long-Tailed Macaques (Macaca fascicularis umbrosus)
}

\author{
Jayashree Mazumder ${ }^{1}$ (D) - Stefano S. K. Kaburu ${ }^{2}$
}

Published online: 12 August 2020

C) Springer Science+Business Media, LLC, part of Springer Nature 2020

\section{Correction to: International Journal of Primatology (2020) 41:141-159 https://doi.org/10.1007/s10764-020-00141-y}

The original version of this article unfortunately contained mistakes in Methods and Acknowledgements sections. The changes are shown below:

1. Under "Methods", "Study site and subjects" section, the sentence "Based on the survey, we identified 28-43 individuals, including 4-8 males, 5-8 females, 5-11 subadults, 5-13 juveniles, and 0-4 infants." should be changed to "Based on the survey, we identified 28-44 individuals, including 4-8 males, 5-8 females, 5-11 subadults, 5-13 juveniles, and 0-4 infants."

2. Under "Acknowledgements" section, the sentence "We thank Parth R. Chauhan for his guidance and support during the study and article preparation." should be changed to "We thank Parth R. Chauhan and Manjari Jain for their guidance and support during the study and article preparation."

The authors apologize for these mistakes.

The online version of the original article can be found at https://doi.org/10.1007/s10764-020-00141-y

Jayashree Mazumder

jayashreemazumder@iisermohali.ac.in

1 Humanities and Social Science Department, Indian Institute of Science Education and Research (IISER), Mohali, India

2 Department of Biomedical Science \& Physiology, Faculty of Science \& Engineering, University of Wolverhampton, Wolverhampton, UK 